\title{
The Importance of Serum Uric Acid Levels and Killip Classification in Predicting Prognosis of Acute Myocardial Infarction
}

\author{
Shivakumar B.G. ${ }^{1}$, Shivakumar N. ${ }^{2}$, Siddharth Gosavi3 ${ }^{3}$, Shashank Shastry ${ }^{4}$ \\ 1, 2, 3, 4 Department of General Medicine, Jagadguru Jayadeva Murugarajendra Medical College, \\ Davangere, Karnataka, India.
}

\begin{abstract}
BACKGROUND

The study was conducted in an attempt to correlate serum uric acid levels with Killip class i.e. severity of heart failure in patients with acute myocardial infarction and to assess any influence of serum uric acid levels on predicting prognosis in patients with acute myocardial infarction. Ischaemic heart disease, particularly acute myocardial infarction is one of the leading causes of death across the world accounting for 12.7 $\%$ of global mortality. Low and middle-income countries are facing $80 \%$ of the global burden of ischaemic heart disease death. Since the pathophysiology of acute myocardial infarction is complicated, proper risk stratification is essential for appropriate management and better outcome. Serum uric acid levels (SUA) have been correlated with coronary artery calcification and atherosclerosis. High SUA levels also have been identified as a risk marker for cardiovascular disease development, progression and mortality.
\end{abstract}

\section{METHODS}

The study design was a one-year cross-sectional study. 100 patients admitted with acute myocardial infarction within one day of the start of symptoms in the Department of Cardiology \& Medicine were included from September 2018 to September 2019. In this study, patients with known causes of elevated uric acid levels (chronic kidney disease, gout, haematological malignancy, hypothyroidism, metabolic syndrome, myeloproliferative disease, lymphoproliferative disease, drugspyrazinamide, diuretics, ethambutol, ethanol, malignancy, G6PD deficiency and psoriasis) were included. Patients on drugs which raise serum uric acid e.g., salicylates ( $2 \mathrm{gm} / \mathrm{d}$, hydrochlorothiazide, pyrazinamide), and chronic alcoholics were not included. Patients were further subjected to investigations like serum uric acid, ECG, 2D echo and other routine investigations. Urine albumin levels, troponin I, chest $x$-ray, fundoscopy, and fasting lipid profile were done. Investigation reports were analysed with the clinical profile and the data was compiled and appropriate statistical test was applied.

\section{RESULTS}

There were more cases of myocardial infarction above 40 years as compared to below 40 years of age and males (69 \%) were more as compared to females (31\%) with the commonest presentation as chest pain. Majority of the patients had inferior wall myocardial infarction (IWMI) $(40 \%)$ and most $(91 \%)$ of the patients had left ventricular (LV) dysfunction (mild, moderate and severe). More patients with Killip class III and IV had abnormal uric acid levels as compared to class I, and II. Among 27 patients who expired, 23 were in Killip class III and IV (13 in Killip class III and 10 in class IV) and the mean serum uric acid levels of expired patients were elevated on all the 3 days with maximum elevation on day 1.

\section{CONCLUSIONS}

Patients with higher Killip class had higher levels of serum uric acid in comparison to patients of lower Killip class. Serum uric acid level in association with Killip class is a good predictor of the severity of heart failure and short-term mortality after myocardial infarction.

\section{KEY WORDS}

Hypertension, Killip Class, Mortality, Serum Uric Acid, Myocardial Infarction, Left Ventricular Dysfunction
Corresponding Author:

Dr. Siddharth Gosavi,

Department of General Medicine,

Medical College Road, MCC B Block,

Kuvempu Nagar, Davangere - 577004,

Karnataka, India.

E-mail: ramshyamsid@gmail.com

DOI: $10.14260 /$ jemds/2021/91

How to Cite This Article:

Shivakumar BG, Shivakumar N, Gosavi S, et al. The importance of serum uric acid levels and killip classification in predicting prognosis of acute myocardial infarction. $J$ Evolution Med Dent Sci 2021;10(07):409413, DOI: $10.14260 /$ jemds/2021/91

Submission 12-09-2020,

Peer Review 16-12-2020,

Acceptance 24-12-2020,

Published 15-02-2021.

Copyright (C) 2021 Shivakumar B.G. et al. This is an open access article distributed under Creative Commons Attribution License [Attribution 4.0 International (CC BY 4.0)] 


\section{BACKGROUND}

Ischaemic heart disease is a condition in which there is an inadequate supply of blood and oxygen to the myocardium, it occurs due to an imbalance between myocardial oxygen supply and demand. Atherosclerosis is the most common cause. Genetic factors, an energy-rich and high-fat diet, smoking and a sedentary lifestyle are associated with the emergence of ischaemic heart disease (IHD). Obesity, insulin resistance and type 2 diabetes mellitus are increasing and are strong risk factors for IHD.

As a result, the prevalence of risk factors for IHD are increasing rapidly in those regions such that a majority of the global burden of IHD occurs there. IHD is likely to become the most common cause of death worldwide by 2020.1 Cardiovascular diseases have been gaining importance in developing countries like India recently because of increased incidence of the disease. It is the first among top 5 causes of deaths in the Indian population (rural vs. urban, economically backward vs. economically developed, men vs. women and at all ages vs. middle age). ${ }^{2}$ Left ventricular dysfunction is the single most important predictor of mortality after acute ST segment elevation myocardial infarction (STEMI).3,4 In 1967, Killip and Kimball ${ }^{5}$ proposed a classification scheme based on the presence and severity of rales detected in patients presenting with STEMI. Killip classification is an important predictor of mortality in patients with acute coronary syndromes. ${ }^{6}$ Some markers indicate unfavourable prognosis in patients with acute myocardial infarction. Serum uric acid is one of the markers that has been evaluated in research.

The role of uric acid as a prognostic marker in cardiovascular syndromes is still controversial. Uric acid is produced by the activity of the enzyme xanthine oxidase and is the final product of purine metabolism. ${ }^{7}$ Xanthine oxidase produces oxidants in this process that may have an important role in cardiovascular disease. Some studies suggest that uric acid implicates inflammation and intracellular stress leading to endothelial injury and enhancement of vasoconstrictor effects. ${ }^{8}$ According to the Japanese Acute Coronary Syndrome Study, ${ }^{9}$ there was a strong correlation between serum uric acid concentration and Killip classification in patients of acute myocardial infarction. Patients who had high uric acid concentrations developed short-term adverse events. Though numerous cardiac biomarkers are available, occasionally there may be a need to find a simple \& reliable prognostic marker in developing countries where fibrinolytic therapy is still the primary mode of management.

We undertook this study to note the levels of serum uric acid in acute myocardial infarction, to correlate the serum uric acid levels with Killip classification in predicting the prognosis in these patients.

\section{METHODS}

The present study was conducted in the Department of General Medicine. Patients attending the hospitals attached to JJM Medical College Bapuji Hospital, Davangere \& Chigateri General Hospital.
The study design was a one-year cross-sectional study. 100 patients with acute myocardial infarction were included in the study. The study was approved by institutional ethics committee and informed consent was obtained.

\section{Inclusion Criteria}

Patients more than 18 years of age, ECG findings and biochemical markers suggestive of acute myocardial infarction. Patients with ST-segment elevation myocardial infarction (STEMI) and non-ST segment elevation myocardial infarction (NSTEMI) were be included in the study.

\section{Exclusion Criteria}

Known causes of elevated uric acid level (chronic kidney disease, gout, haematological malignancy, hypothyroidism, metabolic syndrome, myeloproliferative disease, Lymphoproliferative disease, drugs-pyrazinamide, diuretics, ethambutol, ethanol, malignancy, G6PD deficiency, psoriasis). Patients on drugs which increase serum uric acid e.g. salicylates ( $2 \mathrm{gm} / \mathrm{d}$, hydrochlorothiazide, pyrazinamide).

Patients were interviewed and demographic data, history of present illness, other comorbid conditions, personal history were obtained. Further, these patients underwent clinical examination followed by a systemic examination. The patients who fulfilled the selection criteria were informed about the nature of the study in detail and written informed consent was obtained. Patients were then classified according to clinical signs into Killip Classes.

Investigations carried out were complete blood count, serum uric acid on day 0,3 \& day 5 fasting lipid profile. 12-Lead ECG, CPK-MB, Trop-I (as and when required). 2D ECHO, fasting blood sugar HbA1C were done.

\section{Statistical Analysis}

Statistical Package for Social Sciences (SPSS) version 20 was used to perform the statistical analysis. Descriptive statistics of the explanatory and outcome variables were calculated by the mean, standard deviation for quantitative variables, frequency and proportions for qualitative variables. Inferential statistics like the chi-square test was used to test the significance between qualitative variables unpaired t-test was applied to check the statistical difference between quantitative variables.

\section{RESULTS}

Age-wise distribution of subjects was done in this study. Males (69\%) outnumbered females (31\%) in the present study. Chest pain and dyspnoea was present in $93 \%$ and $52 \%$ of the study subjects. The most common habit present in the study subjects was tobacco (41\%). $31 \%$ of study subjects were smokers. The most common ECG finding seen was IWMI in 40 $\%$, followed by anterior wall myocardial infarction (AWMI) in $28 \%$, and anterolateral myocardial infarction (ALMI) in $12 \%$. LV dysfunction was mild in $50 \%$ of the study subjects. It was found to be severe and moderate in $22 \%$ and $19 \%$ 
respectively. Only $9 \%$ had normal findings. According to Killip classification, the majority belonged to Killip class I (42\%), followed by $22 \%$ in Killip class III, $20 \%$ in Killip class II and $16 \%$ in Killip class IV. In the present study, $27 \%$ of cases passed away whereas $73 \%$ recovered.

Among the expired cases, majority of them belonged to Killip class III (48.1\%), followed by class IV (37\%), class II (11.1\%) and class I (3.7\%). There was a statistically significant association found between Killip classification and the outcome. $(\mathrm{p}=0.001)$. (Table 1) Among the total expired cases, the majority had AWMI (35.71\%), followed by ALMI (33.3\%), IWMI (27.5\%), left atrial myocardial infarction (LAMI) (20 \%), non-ST-segment elevation myocardial infarction (NSTEMI) (16.7\%). There was no significant association found between ECG and outcome. ( $p=0.762)$.

\begin{tabular}{|c|c|c|c|c|c|}
\hline \multirow{2}{*}{ Killip } & \multicolumn{2}{|c|}{ Outcome } & \multirow{2}{*}{ Total } & \multirow{2}{*}{ Chi-Square } & \multirow{2}{*}{ P-Value } \\
\hline & Discharge & Expired & & & \\
\hline \multirow{2}{*}{ I } & 41 & 1 & 42 & \multirow{10}{*}{36.102} & \multirow{10}{*}{0.001} \\
\hline & $56.2 \%$ & $3.7 \%$ & $42.0 \%$ & & \\
\hline \multirow{2}{*}{ II } & 17 & 3 & 20 & & \\
\hline & $23.3 \%$ & $11.1 \%$ & $20.0 \%$ & & \\
\hline \multirow{2}{*}{ III } & 9 & 13 & 22 & & \\
\hline & $12.3 \%$ & $48.1 \%$ & $22.0 \%$ & & \\
\hline \multirow{2}{*}{ IV } & 6 & 10 & 16 & & \\
\hline & $8.2 \%$ & $37.0 \%$ & $16.0 \%$ & & \\
\hline \multirow{2}{*}{ Total } & 73 & 27 & 100 & & \\
\hline & $100.0 \%$ & $100.0 \%$ & $100.0 \%$ & & \\
\hline
\end{tabular}

Age with Killip class comparison revealed that in the age group of up to 60 years a maximum number of patients were in Killip class I and II as whereas age group more than 60 years belonged to class III and IV. There was a significant association found between age and Killip classification. $(p=0.038)$. (Table 2).

\begin{tabular}{|lccccc|}
\hline $\begin{array}{c}\text { Age } \\
\text { (Years) }\end{array}$ & I & II & III & IV & Total \\
\hline$<40$ & 3 & 1 & 1 & 3 & $\mathbf{8}$ \\
$41-60$ & 27 & 14 & 7 & 6 & $\mathbf{5 4}$ \\
$>\quad 60$ & 12 & 5 & 14 & 7 & $\mathbf{3 8}$ \\
Chi-square & \multicolumn{5}{c}{} \\
p Value & 0.038 \\
\hline \multicolumn{7}{|c|}{ Table 2. Cross-Tabulation of Age and Killip Classification } \\
\hline
\end{tabular}

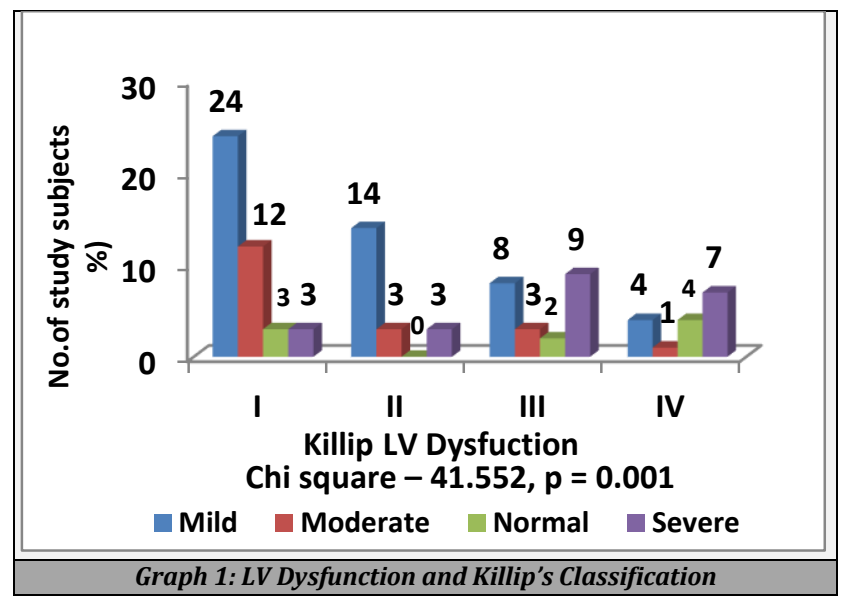

Majority of the study subjects had ECG changes of IWMI with Killip I, followed by AWMI with Killip I and IWMI with Killip III. There was no statistical significance associated with ECG changes and Killip classification ( $p=0.708)$

Majority of the study subjects had a mild grade of LV dysfunction with Killip class I, followed by Killip class II and moderate grade of LV dysfunction with Killip class I. There was a statistically significant association found between LV dysfunction changes and Killip classification $(\mathrm{p}=0.001)$ (Graph 1)

The mean normal and abnormal uric acid on day 0 was $5.791 \pm 0.8878 \mathrm{mg} / \mathrm{dL}$ and $7.461 \pm 0.4452 \mathrm{mg} / \mathrm{dL}$ respectively. The mean normal and abnormal uric acid on day 3 was $5.778 \pm 0.8116 \mathrm{mg} / \mathrm{dL}$ and $7.4 \pm 0.4718 \mathrm{mg} / \mathrm{dL}$ respectively. The mean normal and abnormal uric acid on day 5 was $5.911 \pm 0.6994 \mathrm{mg} / \mathrm{dL}$ and $7.279 \pm 0.3095 \mathrm{mg} / \mathrm{dL}$ respectively. The mean uric acid in males and females on day 0 was $6.688 \pm 1.0775 \mathrm{mg} / \mathrm{dL}$ and $6.165 \pm 1.0923 \mathrm{mg} / \mathrm{dL}$ respectively. Unpaired t-test showed a significant difference between the mean uric acid levels on day 0 and the gender. $(\mathrm{p}=0.027)$. The mean uric acid in males and females on day 3 was $6.588 \pm 1.0116 \mathrm{mg} / \mathrm{dL}$ and $6.068 \pm 1.0842 \mathrm{mg} / \mathrm{dL}$ respectively. Unpaired $t$-test showed a significant difference between the mean uric acid levels on day 3 and the gender. ( $p$ $=0.022$ ).

The mean uric acid in males and females on day 5 was $6.567 \pm 0.8196 \mathrm{mg} / \mathrm{dL}$ and $6.129 \pm 0.9606 \mathrm{mg} / \mathrm{dL}$ respectively. Unpaired t-test showed a significant difference between the mean uric acid levels on day 5 and the gender. ( $p$ $=0.021$ ).

The mean uric acid among discharged and expired on day 0 was $6.303 \pm 1.0973 \mathrm{mg} / \mathrm{dL}$ and $7.130 \pm 0.8866 \mathrm{mg} / \mathrm{dL}$ respectively. Unpaired t-test showed a significant difference between the mean uric acid levels on day 0 and the outcome. $(\mathrm{p}=0.001)$ (Table 3$)$

\begin{tabular}{|c|c|c|cccc|}
\hline $\begin{array}{c}\text { Uric } \\
\text { Acid } \\
\text { (mg / } \\
\text { dL) }\end{array}$ & Gender & N & Mean & S.D. & t-Test & $\begin{array}{c}\text { P- } \\
\text { Value }\end{array}$ \\
Day 0 & Male & 69 & 6.688 & 1.0775 & 2.239 & 0.027 \\
& Female & 31 & 6.165 & 1.0923 & & \\
Day 3 & Male & 69 & 6.588 & 1.0116 & 2.328 & 0.022 \\
& Female & 31 & 6.068 & 1.0842 & & \\
Day 5 & Male & 69 & 6.567 & .8196 & 2.339 & 0.021 \\
\hline \multicolumn{7}{c}{ Table 3. Comparison of Uric Acid Levels with Gender } \\
\hline
\end{tabular}

The mean uric acid among discharged and expired on day 3 was $6.314 \pm 1.0265 \mathrm{mg} / \mathrm{dL}$ and $6.733 \pm 1.0975 \mathrm{mg} / \mathrm{dL}$ respectively. Unpaired t-test showed no significant difference between the mean uric acid levels on day 3 and the outcome. $(\mathrm{p}=0.078)$.

The mean uric acid among discharged and expired on day 5 was $6.285 \pm 0.8545 \mathrm{mg} / \mathrm{dL}$ and $6.826 \pm 0.8574 \mathrm{mg} / \mathrm{dL}$ respectively. Unpaired t-test showed a significant difference between the mean uric acid levels on day 5 and the outcome. (p=0.006) (Table 3)

On day 0 , abnormal uric acid was seen high in study subjects having Killip class III (38.6\%), followed by $27.3 \%$ with Killip class II, $20.5 \%$ with Killip class IV and $13.6 \%$ with Killip class I. Chi-square test showed a significant association between Killip classification and uric acid levels on day $0 .(p=$ 0.001) (Table 4)

Abnormal uric acid levels on day 3 were observed with Killip class III (30 \%), followed by Killip class II (27.5\%), Killip class IV (25 \%) and Killip class I (17.5 \%). Chi-square test 
showed a significant association between Killip classification and uric acid levels on day $3 .(p=0.001)$ (Table 4).

\begin{tabular}{|ccccccc|}
\hline Uric & Outcome & N & Mean & $\begin{array}{c}\text { Std. } \\
\text { Deviation }\end{array}$ & t-Test & P-Value \\
Day 0 & Discharge & 73 & 6.303 & 1.0973 & -3.511 & .001 \\
& Death & 27 & 7.130 & .8866 & & \\
Day 3 & Discharge & 73 & 6.314 & 1.0265 & -1.781 & 0.078 \\
& Death & 27 & 6.733 & 1.0975 & & \multirow{2}{*}{0.006} \\
Day 5 & Discharge & 73 & 6.285 & 0.8545 & -2.808 & \\
& Death & 27 & 6.826 & 0.8574 & \\
\hline \multicolumn{7}{c}{ Table 4. Comparison of Uric Acid Levels with Outcome } \\
\hline
\end{tabular}

Among the study subjects who had abnormal levels of uric acid on day 5 , the majority belonged to Killip class III (36.8 \%), followed by Killip class II (26.3 \%), Killip class IV (23.7\%) and Killip class I (13.2\%). Chi-square test showed a significant association between Killip classification and uric acid levels on day $5 .(p=0.001)$ Table 4 .

The mean uric acid level on day 0 with Killip class I, II, III and IV was $5.86+0.968,6.765+1.0883,7.245+0.7008$ and $6.988+0.9926 \mathrm{mg} / \mathrm{dL}$ respectively.

The mean uric acid level on day 3 with Killip class I, II, III and IV was $5.879+0.9812,6.655+0.9151,6.791+0.8017$ and $7.081+1.1059 \mathrm{mg} / \mathrm{dL}$ respectively.

The mean uric acid level on day 5 with Killip class I, II, III and IV was $5.995+0.8468,6.56+0.75,6.725+0.8745$ and $6.431+0.8845 \mathrm{mg} / \mathrm{dL}$ respectively.

\section{DISCUSSION}

In the present study of 100 patients with acute MI, the importance of serum uric acid levels and Killip class was studied to know about the prognosis in these patients. In our study majority of patients were in Killip class I (42\%), class II (20\%), class III (22\%) and only $16 \%$ in class IV. In our study $73(73 \%)$ patients were discharged after 5 days and 27 (27\%) died during the hospital stay. The outcome of patients in relation with Killip class was done by us and we found that the maximum number of patients who died belonged to class III and IV (13 in class III and 10 class IV) and those who survived, were maximum in Killip class I and II. This shows a positive correlation between mortality and Killip class which is similar to the study by Nadkar et al. ${ }^{10}$ and Shetty et al. ${ }^{11}$

All patients were subjected to echocardiography and it was observed that 9 patients (9\%) had normal left ventricle function and remaining 91 patients $(91 \%)$ had LV dysfunction ranging from mild, moderate to severe. Majority of the study subjects had a mild grade of LV dysfunction with Killip class I, followed by Killip class II and moderate grade of LV dysfunction with Killip class I. There was a statistically significant association found between LV dysfunction changes and Killip classification ( $\mathrm{p}=0.001)$.

The study by Chen et al. ${ }^{12}$ and Shetty et al. ${ }^{11}$ found similar observation when they evaluated LV dysfunction by 2D ECHO and compared with uric acid. Patients with moderate to severe LV dysfunction had high uric acid levels. Serum uric acid estimation revealed that a greater number of patients 44 out of 100 had abnormal levels on day 0, 40 patients on day 3 and 38 patients on day 5 .

The mean normal and abnormal uric acid on day 0 was $5.791 \pm 0.8878 \mathrm{mg} / \mathrm{dL}$ and $7.461 \pm 0.4452 \mathrm{mg} / \mathrm{dL}$ respectively. The mean normal and abnormal uric acid on day 3 was $5.778 \pm 0.8116 \mathrm{mg} / \mathrm{dL}$ and $7.4 \pm 0.4718 \mathrm{mg} / \mathrm{dL}$ respectively. The mean normal and abnormal uric acid on day 5 was $5.911 \pm 0.6994 \mathrm{mg} / \mathrm{dL}$ and $7.279 \pm 0.3095 \mathrm{mg} / \mathrm{dL}$ respectively.

It was statistically not significant. A similar observation was also seen in the study by Nadkar et al. ${ }^{10} \mathrm{We}$ also tried to compare uric acid levels of expired patients; the average uric acid levels of expired patients were raised on all 3 days with the maximum on day 1 . The study by Omidvar et al. ${ }^{13}$ and Car et al. ${ }^{14}$ showed a strong relationship between serum uric acid at the time of admission and in-hospital and short-term mortality. Trkulja V. ${ }^{15}$ found, on admission serum uric acid can predict an outcome. An attempt was made to compare serum uric acid level on day 0, 3 and 5 with different age groups. Abnormal uric acid level on day 0 was found to be high in the age group of $>60$ years (52.3\%), followed by 41 - 60 years $(43.2 \%)$ and $<40$ years $(4.5 \%)$. There was a significant association found between age and uric acid level on day $0 .(p$ $=0.029$ ).

Abnormal uric acid level on day 3 was found to be high in the age group of $>60$ years ( $50 \%$ ), followed by $41-60$ years ( $45 \%)$ and $<40$ years ( $5 \%)$.

There was no significant association found between age and uric acid level on day 3. ( $p=0.118)$. Abnormal uric acid level on day 5 was found to be high in the age group of $41-60$ years ( $52.6 \%)$, followed by $>60$ years $(39.5 \%)$ and $<40$ years (7.9\%). There was no significant association found between age and uric acid level on day 3. ( $p=0.972)$. However, it was statistically not significant. This is similar to the study by PK Sarma ${ }^{16}$ who observed raising trend of serum uric acid as age advances.

Comparing serum uric acid level with gender revealed that abnormal uric acid levels were more in males on day 0 , day 3 and day 5 , however it was statistically not significant. The study by Dharma et al. ${ }^{17}$ also found no significant difference between uric acid levels in both the gender.

An attempt was made to compare serum uric acid levels with Killip class which showed that on all 3 days patient with abnormal uric acid were more in class III. P-value being statistically significant (0.001). Kojima et al., Nadkar et al.,10 Shetty et al. ${ }^{11}$ have observed similar correlation between serum uric acid level and Killip class. In contrast to this, a study by Jularattanaporn et al. observed no association between hyperuricemia and Killip class.

In our study, we tried to correlate serum uric acid level with the outcome of patients, we found a significant correlation between uric acid and mortality, however, it is statistically significant on day 1 and day 5 . Dharma et al. ${ }^{17}$ in their study found adverse cardiovascular outcome with higher serum uric acid level. Omidvar et al. ${ }^{13}$ and Car et al. ${ }^{14}$ observed association between abnormal uric acid level and short-term mortality. Kojima $S$ et al. in their study conducted in japan suggest that hyperuricemia after MI is associated with the development of heart failure. Bickel et al. showed that increased uric acid level is associated with overall mortality.

In our present small sample study of 100 patients, who were evaluated, comparing various variables, we found a significant correlation between higher serum uric acid levels with higher Killip class (III and IV) and when mortality was taken in to account, elevated levels of serum uric acid was seen 
in those patients who expired (27 patients). High uric acid concentrations on admission were strongly associated with adverse clinical outcome in patients who had an acute MI. This simple, easily available, inexpensive laboratory parameter (estimation of serum uric acid) may help in settings where facilities are lacking, can be a useful biomarker when compared with Killip class to prognosticate patients of acute myocardial infarction. To address this issue whether the efficacy of serum uric acid level in these patients is significant or not, a detailed study with large sample size may be required.

\section{CONCLUSIONS}

In patients with acute myocardial infarction, patients with hyperuricemia had higher mortality. There was a positive correlation with elevated serum uric acid levels with Killip class, (P-value being statistically significant).

There was a strong correlation between hyperuricemia and left ventricle dysfunction, (P-value being statistically significant). Hyperuricemia is an indicator of poor prognosis in acute myocardial infarction. Future studies with larger sample size after adjusting comorbid conditions / confounding factors and comparing them with serum uric acid levels and Killip class to know as to whether these factors have a true association or not are required.

Data sharing statement provided by the authors is available with the full text of this article at jemds.com.

Financial or other competing interests: None.

Disclosure forms provided by the authors are available with the full text of this article at jemds.com.

\section{REFERENCES}

[1] Kasper D, Fausi AS, Longo DL. Harrisons principle of internal medicine. 17th edn. New York: McGraw Hill 2005.

[2] Gupta R, Guptha S, Sharma KK, et al. Regional variations in cardiovascular risk factors in India: India heart watch. World J Cardiol 2012;4(4):112-20.

[3] Frisch DR, Giedrimas E, Mohanavelu S, et al. Predicting irreversible left ventricular dysfunction after acute myocardial infarction. Am J Cardiol 2009;103(9):1206-9.

[4] Reynolds HR, Hochman JS. Cardiogenic shock: current concepts and improving outcomes. Circulation 2008;117(5):686-97.

[5] Killip T, Kimball JT. Treatment of myocardial infarction in a coronary care unit. A two-year experience with 250 patients. Am J Cardiol 1967;20(4):457-64.

[6] Khot UN, Jia G, Moliterno DJ, et al. Prognostic importance of physical examination for heart failure in non-STelevation acute coronary syndromes: the enduring value of Killip classification. JAMA 2003;290(16):2174-81.

[7] Wasserman A, Schnell M, Boursi B, et al. Prognostic significance of serum uric acid in patients admitted to the department of medicine. Am J Med Sci 2010;339(1):1521.

[8] Basar N, Sen N, Ozcan F, et al. Elevated serum uric acid predicts angiographic impaired reperfusion and 1-year mortality in ST-segment elevation myocardial infarction patients undergoing percutaneous coronary intervention. J Investig Med 2011;59(6):931-7.

[9] Kojima S, Sakamoto T, Ishihara M, et al. Prognostic usefulness of serum uric acid after acute myocardial infarction (Japanese Acute Coronary Syndrome Study). Am J Cardiol 2005;96(4):489-95.

[10] Nadkar MY, Jain VI. Serum uric acid in acute myocardial infarction. J Assoc Physicians India 2008;56:759-62.

[11] Shetty S, Rao A, Kumar S, et al. Serum uric acid as a prognostic biomarker and its correlation with Killip class in acute myocardial infarction. International Journal of Biomedical Research 2013;4(7):312-6.

[12] Chen L. Serum uric acid in patients with acute STelevation myocardial infarction. World J Emerg Med 2012;3(1):35-9.

[13] Omidvar B, Ayatollahi F, Alasti M. The prognostic role of serum uric acid level in patients with acute ST elevation myocardial infarction. J Saudi Heart Assoc 2012;24(2):738.

[14] Car S, Trkulja V. Higher serum uric acid on admission is associated with higher short-term mortality and poorer long-term survival after myocardial infarction: retrospective prognostic study. Croat Med J 2009;50(6):559-66.

[15] Trkulja V, Car S. On-admission serum uric acid predicts outcomes after acute myocardial infarction: systematic review and meta-analysis of prognostic studies. Croat Med J 2012;53(2):162-72.

[16] Sarma PK, Bari MS, Ahmed AF, et al. Risk of coronary heart disease with raised serum uric acid. Dinajpur Med Col J 2008;1(1):18-20.

Dharma S, Siswanto BB, Soerianata S, et al. Serum uric acid as an independent predictor of cardiovascular event in patients with acute ST-elevation myocardial infarction. J Clinic Experiment Cardiol 2012:S5:005. 